\title{
On dissipation, interfacial energy and size effects in shape memory alloys
}

\author{
Stanisław Stupkiewicz ${ }^{a}$ and Henryk Petryk \\ Institute of Fundamental Technological Research (IPPT), Polish Academy of Sciences, Warsaw, Poland
}

\begin{abstract}
This work presents a multiscale approach aimed at the modelling of evolution of martensitic microstructures with account for the interfacial energy effects. The total Helmholz free energy and the energy dissipated in the system are split into contributions from the bulk material, from phase interfaces and from other boundaries. Microstructure evolution is then determined by the incremental energy minimization. As an application, stress-induced transformation is considered which proceeds by formation and growth of internally twinned martensite plates within the austenite matrix. The interfacial energy is examined at three scales, namely at twin boundaries, austenite-martensite interfaces and at grain boundaries. Both atomic-scale and the elastic micro-strain interfacial energies are included and respective estimates are taken from the materials science literature or predicted using micromechanical finite element analysis. Examples are provided for the cubic-to-orthorhombic transformation in a CuAlNi shape memory alloy. They illustrate the effect of grain size on the macroscopic stress-strain response including the hysteresis width.
\end{abstract}

\section{Introduction}

Martensitic transformation in shape memory alloys proceeds by formation and evolution of microstructures which have a multiscale character, cf. Bhattacharya [2]. Evolution of these complex microstructures is associated with creation and annihilation of interfaces at various scales. The aim of this work is to develop a multiscale approach capable of quantitative modelling of the related phenomena with account for interfacial energy effects, including dissipation. This represents an extension of our previous work [15] where evolution of stress-induced microstructures in the form of rank-two laminates has been modelled with no characteristic dimension.

Length scale effects in martensitic microstructures can be introduced by taking into account interfacial energy at different levels, cf. $[4,13,17]$, or by adding an energy penalty term associated with the higher gradients of the deformation, e.g. $[5,12]$. In the present work, we do not use higher-order gradients, rather, we include the elastic micro-strain energy at the interfaces between austenite and twinned martensite, and also at the crystal boundary attained by martensite plates. That energy is estimated numerically by the finite element method applied to lower-scale interactions, cf. [14] where the unit cell of a periodic rank-two laminate is used and energy minimization is performed with respect to free shape parameters. Atomic scale interfacial energy on twin interfaces is estimated from data in the materials science literature. The interfacial energy estimates at three different levels are combined to derive analytic relationships between characteristic dimensions of the rank-two laminate [11]. Evolution of the microstructure is studied by incremental minimization of the sum of the total free energy and dissipation $[1,3,6]$. In this paper we extend previous works by including the size-dependent contribution of the interfaces to both the free energy and dissipation.

\footnotetext{
${ }^{a}$ e-mail: sstupkie@ippt.gov.pl
} 
The interfacial energy is considered at three scales. The atomic-scale energy is included at the twin boundaries within martensite plates, both the atomic-scale and elastic micro-strain energies are accounted for at the interfaces between austenite and martensite plates, and the elastic micro-strain energy is considered at the boundary of a crystal or grain attained by parallel martensite plates. As a result, a prediction of the macroscopic response is obtained and accompanied by estimates of characteristic dimensions of the microstructure. Furthermore, the interplay between bulk and interfacial energies at different scales governs the size effects.

\section{Modelling framework}

\subsection{Incremental energy minimization}

In this work, the incremental energy minimization approach is used to determine the evolution of microstructure; the general features of this approach are described in Petryk [9]. The martensitic transformation is assumed isothermal and quasi-static at the time scale associated with slowly varying external loading. Note that, at faster time scales, the phenomena are intrinsically dynamic and non-isothermal, but application of suitable scale transitions results in isothermal quasi-static evolution with rate-independent dissipation, see Petryk et al. [10] for detailed discussion.

The approach is based on the following minimization problem

$$
\Delta E \rightarrow \min \quad \text { subject to kinematical constraints, }
$$

where $\Delta E$ is the amount of energy to be supplied to the compound thermodynamic system \{deforming body + loading device + heat reservoir $\}$ during a virtual incremental transformation. The prefix $\Delta$ indicates a virtual increment from a given state, which correspond in general to a given increment of an external control parameter.

In the present context, and assuming that no energy is stored in the loading device, which corresponds to kinematic control or zero external loads, the incremental energy $\Delta E$ is equal to

$$
\Delta E=\Delta \Phi+\Delta \mathcal{D}
$$

where $\Phi$ is the total Helmholz free energy and $\Delta \mathcal{D}$ is the increment of dissipated energy, both per unit representative volume.

\subsection{Free energy with interfacial energy contribution}

Considering a martensitic microstructure forming and evolving within a representative volume element, the total Helmholz free energy $\Phi$ is expressed as a sum of a bulk energy contribution $\Phi_{\mathrm{v}}$ and several interfacial energy contributions $\Phi_{k}$,

$$
\Phi=\Phi_{\mathrm{v}}+\Phi_{\mathrm{i}}, \quad \Phi_{\mathrm{i}}=\sum_{k} \Phi_{k}
$$

Each interfacial energy contribution $\Phi_{k}$ originates from the interfacial energy at a specific scale, which is integrated over all interfaces at this scale and divided by the volume of the representative element. Two types of interfacial energy are considered, namely the atomic scale energy and elastic micro-strain energy of microstructured interfaces. The latter energy is in fact a bulk energy at a suitably fine scale, however, it is associated with the interface and upon averaging it is interpreted as an interfacial energy at a higher scale. Moreover, the elastic micro-strain energy depends on the characteristic dimensions of the microstructure, thus the related contribution to the free energy term $\Phi_{k}$ is size-dependent, just like the contribution of the atomic scale energy.

Accordingly, the bulk energy contribution $\Phi_{\mathrm{v}}$ includes the size-independent contributions, i.e., the size-independent elastic strain energy at all scales, as well as the chemical energy of transformation. 


\subsection{Dissipation}

In analogy to the free energy, the dissipation is also assumed to comprise size-independent bulk contribution $\Delta \mathcal{D}_{\mathrm{v}}$ and size-dependent interfacial contributions $\Delta \mathcal{D}_{k}$,

$$
\Delta \mathcal{D}=\Delta \mathcal{D}_{\mathrm{v}}+\mathcal{D}_{\mathrm{i}}, \quad \Delta \mathcal{D}_{\mathrm{i}}=\sum_{k} \Delta \mathcal{D}_{k}
$$

Evolution of microstructure is associated with creation or annihilation of interfaces. The key assumption of the present model is that the negative increments of interfacial energy, typically associated with annihilation of interfaces, can hardly be reverted into the bulk free energy and thus contribute to dissipation. Accordingly, the size-dependent dissipation $\Delta \mathcal{D}_{k}$ is assumed to be equal to a fraction of the interfacial energy release $-\Delta \Phi_{k}>0$,

$$
\Delta \mathcal{D}_{k}=\kappa_{k}\left(-\Delta \Phi_{k}\right)_{+},
$$

where

$$
(\psi)_{+}= \begin{cases}\psi & \text { if } \psi>0, \\ 0 & \text { if } \psi \leq 0,\end{cases}
$$

and $\kappa_{k}$ is a coefficient, $0 \leq \kappa_{k} \leq 1$. In general, $\kappa_{k}$ is expected to be nearly equal to unity at low scales and possibly smaller, but still close to unity, at higher scales.

The size-independent bulk dissipation term $\Delta \mathcal{D}_{\mathrm{v}}$ includes all the remaining dissipation mechanisms and is expressed in terms of the volume fraction $\eta$ of martensite, namely

$$
\Delta \mathcal{D}_{\mathrm{v}}=f_{\mathrm{c}}|\Delta \eta|,
$$

where $f_{\mathrm{c}}>0$ is the threshold value for the thermodynamic driving force $f=\partial \Phi_{\mathrm{v}} / \partial \eta$. When the interfacial energy terms are disregarded, the energy minimization framework with $\Delta \mathcal{D}_{\mathrm{v}}$ given by (7) leads to the transformation criterion $|f| \leq f_{\mathrm{c}}$, used e.g. by Stupkiewicz and Petryk [15].

\section{Size effects in a rank-three laminate}

As an application we consider a laminate formed by grains of two orientations separated by flat, parallel grain boundaries. Within each grain, internally-twinned martensite plates are induced by stress in the austenite matrix, thus the resulting microstructure is a rank-three laminate, cf. Fig. 1. This highly idealized model of a polycrystalline shape memory alloy has been introduced by Petryk et al. [10] with the aim to include interfacial energy at three scales: twin boundaries, austenite-martensite interfaces and grain boundaries. At the same time, an exact micro-macro transition can be performed for this microstructure by sequantially applying the micro-macro transition relationships for simple laminates.

Assuming a special orientation of the grains with respect to the grain boundaries and with respect to the direction of uniaxial tension or compression, the bulk contribution to the free energy can be expressed in the following form [10],

$$
\Phi_{\mathrm{v}}=\phi_{0}+\eta \Delta^{\mathrm{am}} \phi_{0}+\frac{1}{2} E\left(\varepsilon_{\mathrm{u}}-\eta \bar{\varepsilon}^{\mathrm{t}}\right)^{2}+\frac{1}{2} q E \eta^{2}
$$

where $\Delta^{\mathrm{am}} \phi_{0}$ is the so-called chemical energy, $E$ is the Young's modulus (elastic isotropy is assumed), $\varepsilon_{\mathrm{u}}$ is the macroscopic uniaxial strain, $\bar{\varepsilon}^{\mathrm{t}}$ is the uniaxial component of the macroscopic transformation strain, and $q$ is a hardening-like parameter associated with incompatibility of the transformation strains in the neighbouring grains. Note that the expression (8) results from an exact micro-macro transition with the crystallographic features of transformation and three-dimensional stress state fully accounted for.

The interfacial energy is considered at three scales, namely $\gamma_{\mathrm{tw}}$ at twin boundaries, $\gamma_{\mathrm{am}}$ at austenite-martensite interfaces, and $\gamma_{\mathrm{gb}}$ at grain boundaries,

$$
\gamma_{\mathrm{tw}}=\gamma_{\mathrm{tw}}^{\mathrm{a}}, \quad \gamma_{\mathrm{am}}=\gamma_{\mathrm{am}}^{\mathrm{a}}+\gamma_{\mathrm{am}}^{\mathrm{e}}=\gamma_{\mathrm{am}}^{\mathrm{a}}+\Gamma_{\mathrm{am}}^{\mathrm{e}} h_{\mathrm{tw}}, \quad \gamma_{\mathrm{gb}}=\gamma_{\mathrm{gb}}^{\mathrm{e}}=\Gamma_{\mathrm{gb}}^{\mathrm{e}} H .
$$



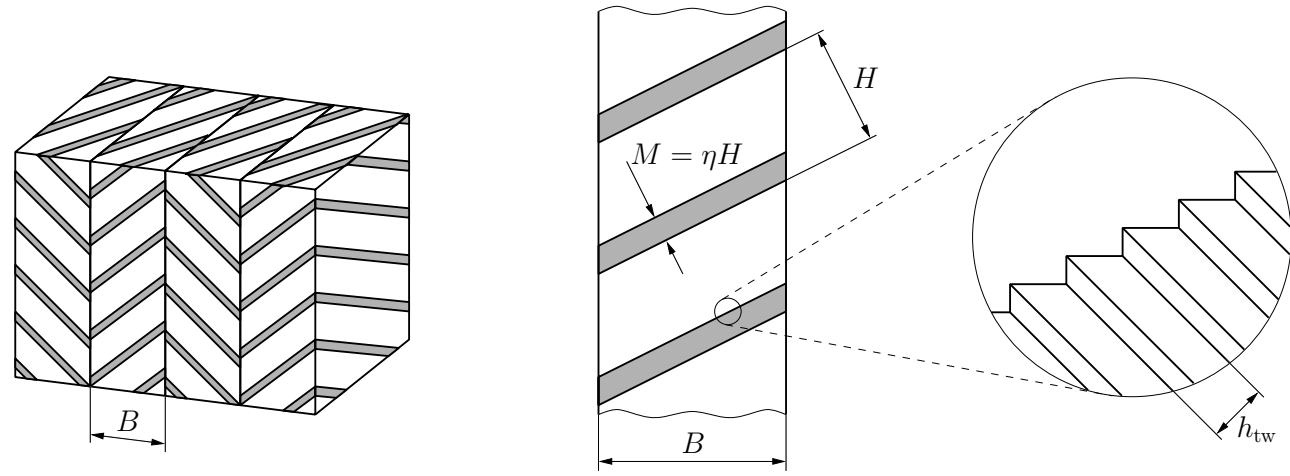

Fig. 1. Idealized SMA polycrystal in the form of a rank-three laminate.

Here the atomic scale energies are denoted by $\gamma_{k}^{\mathrm{a}}$, while $\gamma_{k}^{\mathrm{e}}$ denotes the elastic micro-strain energy which is equal to the product of the size-independent elastic micro-strain energy factor $\Gamma_{k}^{\mathrm{e}}$ and the respective spacing, cf. Fig. 1. Note that the atomic-scale energy of the grain boundary $\gamma_{\mathrm{gb}}^{\mathrm{a}}$ is disregarded here (i.e., effectively, this energy is assumed constant). Also the minor contribution of elastic micro-strains induced by fine twins approaching the grain boundary (within martensite plates) is neglected in $\gamma_{\mathrm{gb}}$.

The elastic micro-strain energy at the austenite-twinned martensite interface, $\gamma_{\mathrm{am}}^{\mathrm{e}}=\Gamma_{\mathrm{am}}^{\mathrm{e}} h_{\mathrm{tw}}$ results from local incompatibility of the austenite and a single variant of martensite (note that the internally-twinned martensite is compatible with the austenite in the average sense only). The interfacial energy factor $\Gamma_{\mathrm{am}}^{\mathrm{e}}$ and the related low energy morphologies of the transition layer at the austenite-twinned martensite interface in $\mathrm{CuAlNi}$ alloy have been determined in $[7$, 14].

Similarly, $\gamma_{\mathrm{gb}}^{\mathrm{e}}=\Gamma_{\mathrm{gb}}^{\mathrm{e}} H$ is the elastic micro-strain energy at the grain boundary approached by martensite plates. The dependence of the energy factor $\Gamma_{\mathrm{gb}}^{\mathrm{e}}$ on the geometrical parameters has been determined in [10] using the finite element method. Furthermore, an approximating function has been introduced in the form

$$
\Gamma_{\mathrm{gb}}^{\mathrm{e}}=\Gamma_{*}^{\mathrm{e}} \mu b^{2}, \quad \Gamma_{*}^{\mathrm{e}}=a \cos \alpha \frac{1-\nu \sin ^{2} \beta}{1-\nu}\left[\eta^{2}\left(1-\sin \frac{\pi \eta}{2}\right)^{2}+(1-\eta)^{2}\left(1-\cos \frac{\pi \eta}{2}\right)^{2}\right]
$$

where $a=0.198$ is a universal coefficient that has been fitted against the finite element results, $b$ is the norm of the shape strain vector $\mathbf{b}$ (i.e. $b$ is the magnitude of the transformation shear strain in the twinned martensite plate), $\mu$ is the shear modulus, $\nu$ is the Poisson's ratio, while angles $\alpha$ and $\beta$ define the geometry of the martensite plates and orientation of the shape strain vector $\mathbf{b}$, respectively.

The total area of the interfaces in a unit reference volume can readily be determined for the considered microstructure in terms of the microstructure parameters $h_{\mathrm{tw}}, H, B$, and $\eta=M / H$. Note that grain orientations satisfy a special symmetry relationship so that the microstructure parameters are identical for both grain orientations. The interfacial part of the total free energy $\Phi_{\mathrm{i}}$ is finally determined as $[11]$

$$
\Phi_{\mathrm{i}}=\Phi_{\mathrm{tw}}+\Phi_{\mathrm{am}}+\Phi_{\mathrm{gb}}
$$

where

$$
\Phi_{\mathrm{tw}}=\frac{2 \eta \gamma_{\mathrm{tw}}^{\mathrm{a}}}{h_{\mathrm{tw}}}, \quad \Phi_{\mathrm{am}}=\frac{2\left(\Gamma_{\mathrm{am}}^{\mathrm{e}} h_{\mathrm{tw}}+\gamma_{\mathrm{am}}^{\mathrm{a}}\right)}{H}, \quad \Phi_{\mathrm{gb}}=\frac{2 \Gamma_{\mathrm{gb}}^{\mathrm{e}} H}{B} .
$$

Below, selected illustrative examples are provided for the CuAlNi shape memory alloy undergoing the cubic-to-orthorhombic $\left(\beta_{1} \rightarrow \gamma_{1}^{\prime}\right)$ transformation. The corresponding parameters of the model are provided in Table 1. 
Table 1. Model parameters used in the numerical examples.

\begin{tabular}{|c|c|c|c|c|c|}
\hline \multicolumn{3}{|c|}{ Material parameters } & \multicolumn{3}{|c|}{ Calculated parameters } \\
\hline$E$ & 100 & $\mathrm{GPa}$ & $\Gamma_{\mathrm{am}}^{\mathrm{e}}$ & 3.2 & $\mathrm{MJ} / \mathrm{m}^{3}$ \\
\hline$\nu$ & 0.3 & & $\bar{\varepsilon}^{\mathrm{t}}$ & 0.0452 & \\
\hline$\Delta^{\mathrm{am}} \phi_{0}$ & 8 & $\mathrm{MJ} / \mathrm{m}^{3}$ & $b$ & 0.0959 & \\
\hline$f_{c}$ & 2 & $\mathrm{MJ} / \mathrm{m}^{3}$ & $\alpha$ & 42.8 & $\operatorname{deg}$ \\
\hline$\gamma_{\mathrm{tw}}^{\mathrm{a}}$ & 0.02 & $\mathrm{~J} / \mathrm{m}^{2}$ & $\beta$ & 22.8 & deg \\
\hline$\gamma_{\mathrm{am}}^{\mathrm{a}}$ & 0.3 & $\mathrm{~J} / \mathrm{m}^{2}$ & $q$ & $7.12 \times 10^{-5}$ & \\
\hline$B$ & $20,60,180$ & $\mu \mathrm{m}$ & & & \\
\hline$\kappa_{\mathrm{tw}}$ & 1 & & & & \\
\hline$\kappa_{\mathrm{am}}$ & 0.95 & & & & \\
\hline$\kappa_{\mathrm{gb}}$ & 0.90 & & & & \\
\hline
\end{tabular}

\subsection{Minimization of free energy (no dissipation)}

As a reference for further considerations let us consider the microstructures that minimize the total free energy (thus dissipation is temporarily disregarded). The total free energy $\Phi$, is the sum of the bulk and interfacial energy terms $\Phi_{\mathrm{v}}$ and $\Phi_{\mathrm{i}}$ specified by (8) and (11), respectively, and depends on the uniaxial strain $\varepsilon_{\mathrm{u}}$, adopted as a control parameter, and three microstructural parameters $\eta, M$ and $h_{\mathrm{tw}}$ (or equivalently $H, M$ and $h_{\mathrm{tw}}$ in view of the relationship $\eta=M / H$ ).

Figure 2 presents sample results obtained for model parameters summarized in Table 1. Microstructure parameters $H^{-1}, M$ and $h_{\mathrm{tw}}$ are shown in Figs. 2(b-d) for three values of the grain size parameter $B$ and the corresponding stress-strain relationships are shown in Fig. 2(a). In all cases, the size effect induced by the interfacial energy is clearly visible. In particular, it is seen that decreasing the grain size results in the refinement of microstructure. At the same time, the associated effect on the stress-strain curve is more pronounced for smaller $B$. This is because finer microstructure carries more interfacial energy.

We remind that energy dissipation is disregarded here. Accordingly, the stress-strain diagrams in Fig. 2(a) (corresponding to the strain control) exhibit no hysteresis. The effect of dissipation on the stress-strain response and on the evolution of microstructure parameters is examined below.

\subsection{Minimization of total incremental energy supply}

Once energy dissipation is accounted for, the process becomes path-dependent and the incremental energy minimization framework is applied, as outlined in Section 2.1. In the present model, we adopt a simplifying assumption that energy dissipation rules out a possible variation of twin spacing $h_{\mathrm{tw}}$ during transformation. Accordingly, we assume that $h_{\mathrm{tw}}$ where $h_{\mathrm{tw}}^{0}$ is the twin spacing that minimizes the total free energy at the initial instant of transformation, i.e. for $\eta=0$.

The effect of grain size $B$ on the pseudoelastic stress-strain diagram is illustrated in Fig. 3 . The dotted line denotes the response corresponding to an infinite grain (i.e. with no interfacial energy). The grain size effect is more pronounced for smaller grains. In particular, the width of hysteresis loop, i.e. energy dissipated in an isothermal process, increases with decreasing grain size. This effect is in a qualitative agreement with experimental observations, e.g. [8]. Note that an inverse relationship is observed in nano-crystalline materials [16], however, this effect is not covered by the present study.

Evolution of microstructure parameters is presented in Fig. 4, and the constant twin spacing $h_{\mathrm{tw}}$ is equal to $47.2,63.9$, and $87.1 \mathrm{~nm}$ for $B$ equal to, respectively, 20,60 , and $180 \mu \mathrm{m}$. The dependence of parameters $H$ and $M$ on the grain size $B$ is qualitatively similar to that shown in Fig. 2, however, they exhibit more complex evolution due to the varying activity of the different dissipation mechanisms. 
(a)

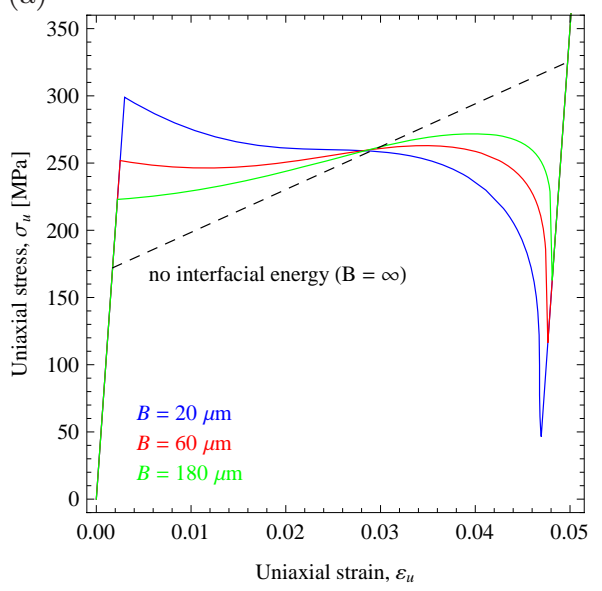

(c)

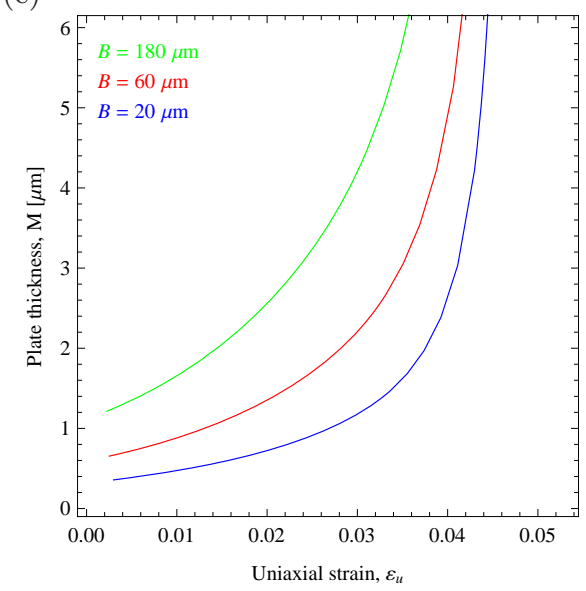

(b)

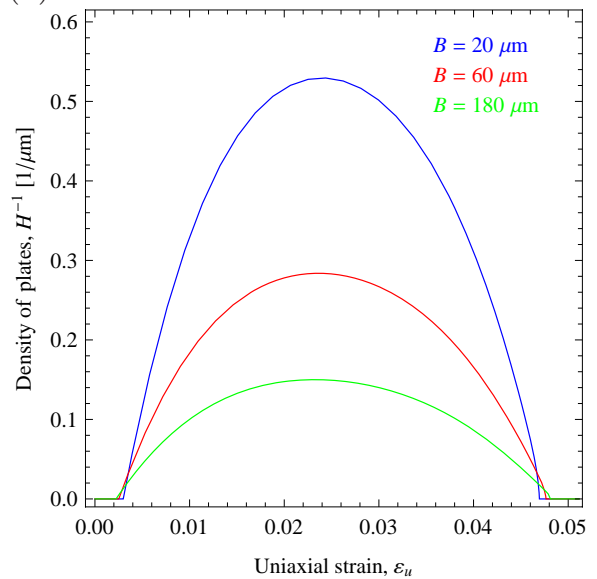

(d)

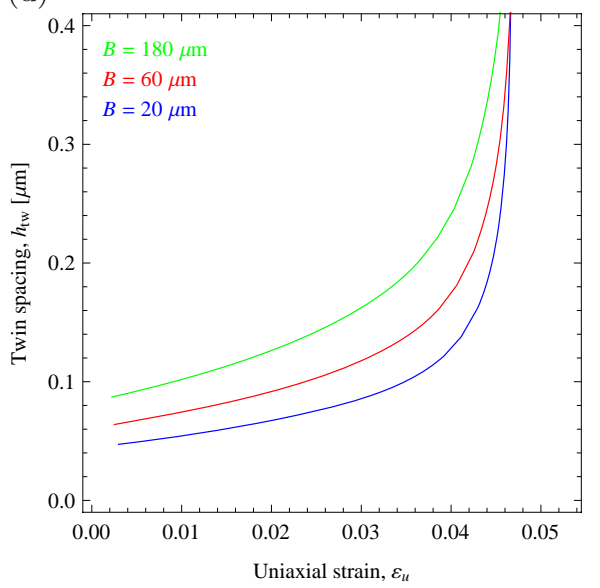

Fig. 2. Minimization of total free energy (no dissipation): (a) stress-strain diagram, (b) density of plates $H^{-1}$, (c) plate thickness $M$, (d) twin spacing $h_{\mathrm{tw}}$.

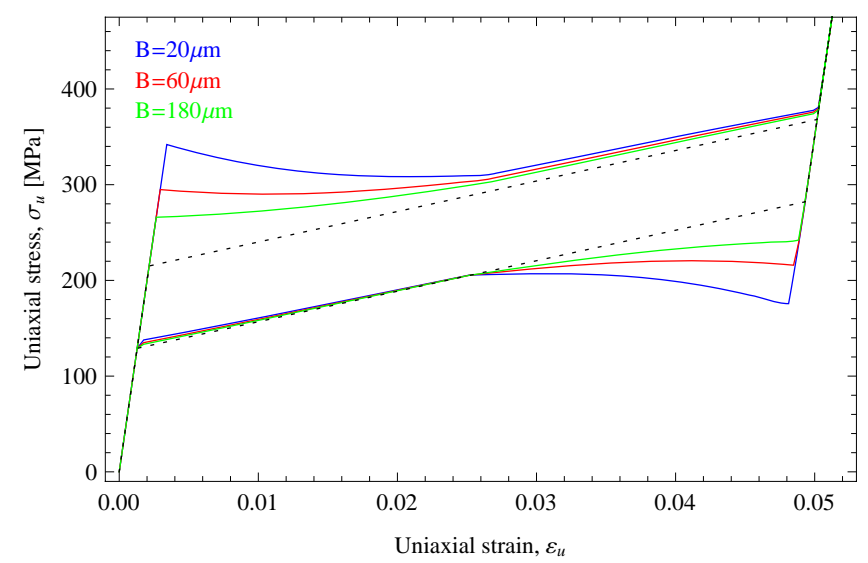

Fig. 3. Stress-strain response during pseudoelastic loading-unloading loop: effect of grain size. 
(a)

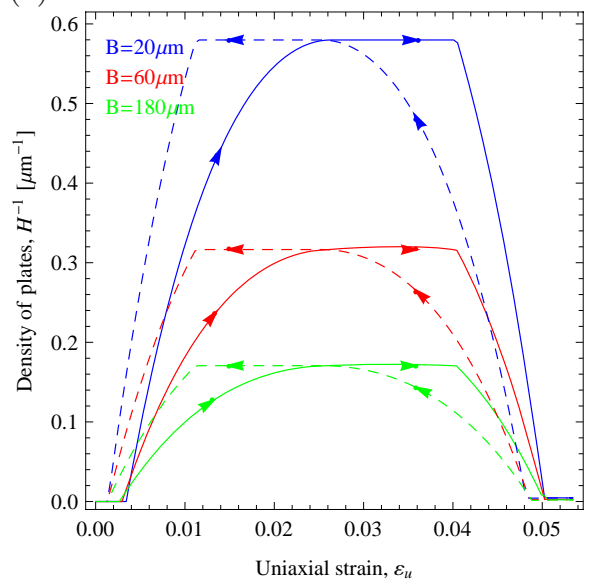

(b)

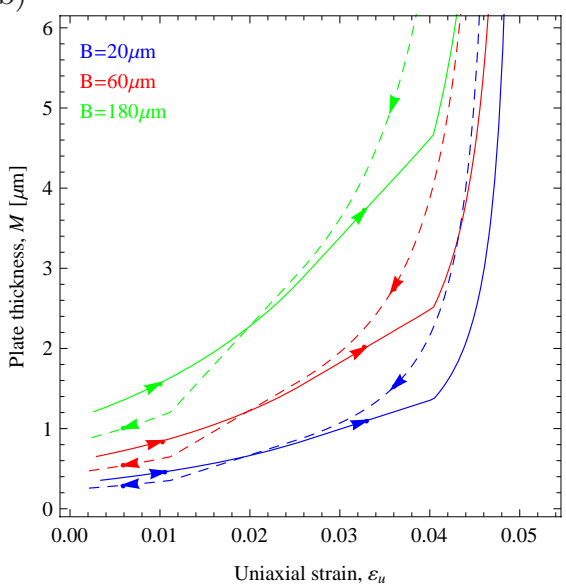

Fig. 4. Effect of grain size on the evolution of microstructure parameters during loading-unloading pseudoelastic loop: (a) density of plates $H^{-1}$, (b) plate thickness $M$ (solid lines - loading, dashed lines - unloading).

\section{Concluding remarks}

1. A micromechanical model of pseudoelastic shape memory alloys has been developed in which the interfacial energy is included into the total free energy. Specifically, the atomicscale and the elastic micro-strain energy is accounted for at three scales: at twin boundaries, austenite-marteniste interfaces and grain boundaries.

2. Negative increments of the interfacial energy at different scales, which are associated with annihilation of interfaces, are assumed assumed to contribute to dissipation.

3. As a result, the model allows quantitative evaluation of size effects without introducing any artificial length-scale parameters. In particular, the characteristic dimensions of the microstructure, such as twin spacing and thickness of martensite plates, are obtained by incremental energy minimization.

4. Effect of grain size of the stress-strain response, hysteresis width, and microstructure parameters has been demonstrated for an idealized polycrystal of CuAlNi shape memory alloy.

\section{Acknowledgment}

This work has been partially supported by the Polish Ministry of Science and Higher Education through Grant No. N N501 267734.

\section{References}

1. M. Arndt, M. Griebel, V. Novak, T. Roubiček, and P. Sittner. Martensitic transformation in NiMnGa single crystals:

Numerical simulation and experiments.

Int. J. Plasticity, 22:1943-1961, 2006.

2. K. Bhattacharya.

Microstructure of martensite: why it forms and how it gives rise to the shape-memory effect.

Oxford University Press, Oxford, 2003.

3. K. Hackl and R. Heinen.

A micromechanical model for pretextured polycrystalline shape-memory

alloys including elastic anisotropy.

Continuum Mech. Thermodyn., 19:499-510, 2008. 
4. A.G. Khachaturyan.

Theory of Structural Transformations in Solids.

John Wiley and Sons, New York, 1983.

5. R.V. Kohn and S. Müller.

Branching of twins near an austenite-twinned-martensite interface. Phil. Mag. A, 66(5):697-715, 1992.

6. M. Kružik, A. Mielke, and T. Roubiček.

Modelling of microstructure and its evolution in shape-memory-alloy single-crystals, in particular in CuAlNi.

Meccanica, 40:389-419, 2005.

7. G. Maciejewski, S. Stupkiewicz, and H. Petryk.

Elastic micro-strain energy at the austenite-twinned martensite interface.

Arch. Mech., 57(4):277-297, 2005.

8. S. Montecinos, A. Cuniberti, and A. Sepúlveda.

Grain size and pseudoelastic behaviour of a $\mathrm{Cu}-\mathrm{Al}-\mathrm{Be}$ alloy.

Mater. Char., 59:117-123, 2008.

9. H. Petryk.

Incremental energy minimization in dissipative solids.

C. R. Mecanique, 331:469-474, 2003.

10. H. Petryk, S. Stupkiewicz, and G. Maciejewski.

Interfacial energy and dissipation in martensitic phase

transformations.

(in preparation).

11. H. Petryk, S. Stupkiewicz, and G. Maciejewski.

Modelling of austenite/martensite laminates with interfacial energy effect.

In P. Tong and Q.P. Sun, editors, Proc. IUTAM Symp. on Size

Effects on Material and Structural Behaviour at Micron- and Nano-scales, pages 151-162. Springer, 2006.

12. X. Ren and L. Truskinovsky.

Finite scale microstructures in nonlocal elasticity.

J. Elasticity, 59:319-355, 2000.

13. A.L. Roytburd.

Thermodynamics of polydomain heterostructures. II. Effect of microstresses.

J. Appl. Phys., 83(1):239-245, 1998.

14. S. Stupkiewicz, G. Maciejewski, and H. Petryk.

Low-energy morphology of the interface layer between austenite and twinned martensite.

Acta Mater., 55:6292-6306, 2007.

15. S. Stupkiewicz and H. Petryk.

Modelling of laminated micro-structures in stress-induced martensitic transformation.

J. Mech. Phys. Solids, 50:2303-2331, 2002.

16. Q.P. Sun and Y.J. He.

A multiscale continuum model of the grain size dependence of the hysteresis in shape memory alloy polycrystals.

Int. J. Sol. Struct., 45:3868-3896, 2008.

17. T. Waitz, T. Antretter, F.D. Fischer, N.K. Simha, and H.P. Karnthaler. Size effects on the martensitic phase transformation of NiTi nanograins.

J. Mech. Phys. Solids, 55:419-444, 2007. 